\title{
Degradación y Mineralización de Tartrazina mediante Electro- oxidación. Optimización de las Condiciones de Operación
}

\author{
Edison GilPavas ${ }^{(1)}$, Izabela Dobrosz-Gómez ${ }^{(2)}$ y Miguel Á. Gómez-García ${ }^{(3)}$ \\ (1) Universidad EAFIT, Departamento de Ingeniería de Procesos. GIPAB: Cr 49 \#7 sur 50, \\ Medellín-Colombia (e-mail: egil@eafit.edu.co) \\ (2) Universidad Nacional de Colombia, Departamento de Física y Química, Sede Manizales, PRISMA: Cra. \\ 2764 -60, A. A. 127, Manizales-Colombia (e-mail: idobrosz-gomez@unal.edu.co) \\ (3) Universidad Nacional de Colombia, Departamento de Ingeniería Química, Sede Manizales, PRISMA: \\ Cra. 2764 -60, A. A. 127, Manizales-Colombia (e-mail: magomez@unal.edu.co)
}

Recibido Dic. 16, 2013; Aceptado Feb. 20, 2014; Versión final recibida Mar. 6, 2014

\begin{abstract}
Resumen
Este trabajo presenta los resultados de la optimización del proceso de electro-oxidación (EO) de la tartrazina. El reactor consta de dos electrodos: uno de diamante dopado con boro y otro de titanio, en configuración monopolar. Se establecieron las variables más significativas del proceso: la concentración inicial del colorante (Ci), la densidad de corriente (i) y el pH. Un diseño experimental determinó sus valores óptimos: $\mathrm{Ci}=30 \mathrm{ppm}, \mathrm{i}=5 \mathrm{~mA} / \mathrm{cm}^{2}$ y $\mathrm{pH}=6.0$. A estas condiciones, se caracterizó la cinética del proceso en términos de: el porcentaje de degradación de color (\%DC), la demanda química de oxígeno (\%DDQO) y la disminución del carbono orgánico total (\%DCOT). Adicionalmente, se evaluó el efecto de la presencia de $\mathrm{Fe}^{2+}$ (proceso electro-Fenton, EF) y Fe ${ }^{2+} / \mathrm{UV}$ (proceso electro-foto-Fenton, EFF). El trabajo demuestra la hipótesis sobre cuáles son los parámetros más significativos en el proceso EO.
\end{abstract}

\section{Tartrazine Degradation and Mineralization by Electro- oxidation. Optimization of the Operating Conditions}

\begin{abstract}
In this work, the operational conditions of the tartrazine electro-oxidation (EO) process were optimized. The batch reactor used has two electrodes: one made of diamond doped with boron and the other one of titanium, working at monopolar configuration. The initial dyestuff concentration (Ci), the current density (i) and the $\mathrm{pH}$ were defined as the main factors affecting the EO. Their optimal values were found as follows: $\mathrm{Ci}=30 \mathrm{ppm}, \mathrm{i}=5 \mathrm{~mA} / \mathrm{cm}^{2}$ and $\mathrm{pH}=6.0$. At these conditions, a kinetic analysis was performed in the terms of: the percentage of the dyestuff decolorization $(\% \mathrm{DC})$, the percentage of the chemical oxygen demand (\%DCOD), and the percentage of the total organic carbon (\%TOC). Additionally, the effect of $\mathrm{Fe}^{2+}$ (electroFenton process, EF) and $\mathrm{Fe}^{2+} / \mathrm{UV}$ radiation (electro-photo-Fenton process, EFF) on the studied process were evaluated. The work demonstrates the validity of the hypothesis about the most significant parameters that affect the EO process.
\end{abstract}

Keywords: tartrazine, electro-oxidation, electro-Fenton, electro-photo-Fenton, optimization 


\section{INTRODUCCIÓN}

Las aguas residuales industriales que contienen colorantes son complejas de tratar. Los colorantes aparecen en los efluentes de la fabricación de papel y pulpa, teñido de tela, textiles, tratamiento de cuero, impresión, productos alimenticios y de tinte. En la actualidad, existen más de 10000 colorantes comercialmente disponibles (Wawrkzkiewics y Hubicki, 2009) y se estima que entre el 10 y el $15 \%$ del colorante se pierde en el efluente durante el proceso. Estos valores son preocupantes si se considera que los colorantes pueden ser micro-tóxicos y cancerígenos para vida acuática (Gupta et al., 2011). Además, las operaciones industriales de coloración exigen gran cantidad de agua, entre 125 y $170 \mathrm{~L} / \mathrm{kg}$ de producto (Robinson et al., 2001). Las estructuras de los colorantes y sus atributos son muy complejos y variables, muchos de ellos tienen origen orgánico, son solubles en el agua, tienen alta resistencia a la acción de agentes químicos, así como baja biodegradabilidad (GilPavas, et al., 2012). La mayoría de estos residuos (aproximadamente el $70 \%$ en peso) son del tipo azo, mientras que los colorantes de antraquinona constituyen la segunda clase más importante de los colorantes textiles (Modirshahla, Behnajady y Kooshaiian, 2007; Yusuf y Reza, 2012).

La tartrazina es un colorante azoico, sintético, amarillo, utilizado a menudo en la industria textil, de alimentos y farmacéutica (Modirshahla, Behnajady y Kooshaiian, 2007). Convencionalmente, las aguas residuales contaminadas por colorantes azoicos se tratan con métodos de precipitación o adsorción seguidos, ocasionalmente, por tratamientos biológicos. Estos métodos presentan importantes problemas relacionados con la generación de lodos peligrosos difíciles de eliminar (Cruz et al, 2010). Un ejemplo de esto es la formación de aminas aromáticas en procesos de tratamiento anaerobios, que pueden ser más tóxicas que la molécula del colorante mismo (Jonstrup, Punzi y Mattiasson, 2011; Yusuf y Reza, 2012). Recientemente, los procesos avanzados de oxidación (PAO) se han convertido en una alternativa interesante para el tratamiento de los colorantes azo. En uno de estos procesos, el proceso de electro-oxidación (EO), el reactivo principal es un electrón que se encarga de degradar los compuestos orgánicos presentes en el efluente (Mohan, Balasubramanian, y Basha, 2007). Fundamentalmente, los métodos electroquímicos están constituidos por dos medios diferentes: el primero ocurre en el ánodo, en donde la oxidación se produce en la superficie del electrodo (electrólisis directa), y el segundo, cuando la oxidación se produce continuamente en la solución a través del mediador en la superficie anódica (electrólisis indirecta) (GilPavas et al., 2009; Mohan, Balasubramanian, y Basha, 2007). En la electrólisis directa, la velocidad de oxidación depende de la actividad del electrodo, la velocidad de difusión de los contaminantes y de la densidad de corriente. Por el otro lado, la temperatura, el $\mathrm{pH}$ y la velocidad de difusión de los oxidantes generados determinan la velocidad de oxidación en la electrólisis indirecta (Martínez-Huitle y Brillas, 2009). En esta última, es una práctica común adicionar sales como el $\mathrm{NaCl}$ o el $\mathrm{KCl}$ a las aguas residuales para mejorar su conductividad y para generar iones hipoclorito beneficiosos para la degradación.

Otros procesos de EO se basan en la producción continúa in situ de $\mathrm{H}_{2} \mathrm{O}_{2}$ mediante el dopaje de los electrodos de diamante de la unidad de electrólisis y la reducción de dos electrones del $\mathrm{O}_{2}$ inyectado al sistema, como se muestra en la reacción (1) (Brillas et al., 2010; Badellino, Arruda y Bertazzoli, 2006).

$$
\mathrm{O}_{2}+2 \mathrm{H}^{+}+2 e^{-} \rightarrow \mathrm{H}_{2} \mathrm{O}_{2}
$$

De esta forma, es posible aumentar el grado de mineralización de los contaminantes orgánicos con costos energéticos relativamente bajos. De hecho, el gran poder oxidante de los electrodos de diamante dopados con boro (DDB) para eliminar contaminantes orgánicos se atribuye a su alto sobrepotencial de $\mathrm{O}_{2}$. Este permite la generación de gran cantidad de radicales ${ }^{\circ} \mathrm{OH}\left(\mathrm{DDB}\left({ }^{\circ} \mathrm{OH}\right)\right)$ adsorbidos sobre su superficie tras la oxidación del agua, como se representa en la ecuación (2), y la posterior oxidación de la materia orgánica de acuerdo a la ecuación (3) (Brillas et al., 2010; Patiño et al., 2012).

$$
\mathrm{DDB}+\mathrm{H}_{2} \mathrm{O} \rightarrow \mathrm{DDB}\left({ }^{\bullet} \mathrm{OH}\right)_{\mathrm{Ads}}+\mathrm{H}^{+}+e^{-}
$$

$$
\mathrm{DDB}\left({ }^{\bullet} \mathrm{OH}\right)_{\mathrm{Ads}}+\mathrm{MO} \rightarrow \mathrm{DDB}+m \mathrm{CO}_{2}+n \mathrm{H}_{2} \mathrm{O}+\mathrm{H}^{+}+e^{-}
$$

El potencial de oxidación del $\mathrm{H}_{2} \mathrm{O}_{2}$ se puede incrementar mediante la adición del ion $\mathrm{Fe}^{2+}$ en medio ácido. Este ayuda a generar los radicales ${ }^{\circ} \mathrm{OH}$ y el ion $\mathrm{Fe}^{3+}$ mediante la reacción Fenton (ecuación (4)) (Esquivel et al., 2009).

$$
\mathrm{Fe}^{2+}+\mathrm{H}_{2} \mathrm{O}_{2} \rightarrow \mathrm{Fe}^{3+}+{ }^{\bullet} \mathrm{OH}+\mathrm{HO}^{-}
$$


Esta variante de la EO es conocida como el proceso electro-Fenton (EF) (Oturan et al., 2010). En el, los radicales ${ }^{\circ} \mathrm{OH}$ producidos se caracterizan por su alto potencial estándar de reducción $\left(\mathrm{E}^{\circ}=2,8 \mathrm{~V}\right.$ vs $\left.\mathrm{SHE}\right)$ (Rocha et al., 2011). Ellos son capaces de mineralizar, de una forma no selectiva, contaminantes orgánicos a $\mathrm{CO}_{2}, \mathrm{H}_{2} \mathrm{O}$ e iones inorgánicos (Flox et al., 2007). Otra ventaja del proceso EF sobre el proceso Fenton clásico es la rápida regeneración del ion $\mathrm{Fe}^{2+}$ por la reducción del ion $\mathrm{Fe}^{+3}$ en el cátodo (Martínez-Huitle y Brillas, 2009). El proceso EF se puede mejorar irradiando la solución con la ayuda de lámparas UV ( $\lambda \max =365 \mathrm{~nm}$ ). Este proceso se conoce como electro-foto-Fenton (EFF). En el, la radiación actúa como catalizador dando lugar tanto a la fotólisis de complejos de $\mathrm{Fe}^{3+}$, con la generación de ácidos carboxílicos, así como a la regeneración del $\mathrm{Fe}^{2+}$ a partir del $\mathrm{Fe}(\mathrm{OH})^{2+}$. Así, la mineralización de los compuestos orgánicos aumenta mediante los radicales ${ }^{\circ} \mathrm{OH}$ generados mediante las ecuaciones (5) y (6) (Flox et al., 2007). Las principales ventajas de las tecnologías de EO, EF y EFF son la simplicidad en su uso, accesibilidad y costo moderado.

$$
\mathrm{Fe}(\mathrm{OH})^{2+}+h v \rightarrow \mathrm{Fe}^{2+}+{ }^{\bullet} \mathrm{OH}
$$

$$
\mathrm{H}_{2} \mathrm{O}_{2}+h v \rightarrow 2^{\circ} \mathrm{OH}
$$

Pocos estudios se han reportado en la literatura relacionados con la degradación y mineralización de la tartrazina. En uno de ellos, Modirshahla et al. (2007) compararon diferentes materiales de electrodos para la decoloración de la tartrazina mediante electrocoagulación. Sus resultados indicaron que las variables que más influyen en la remoción del color fueron la densidad de corriente, el tiempo de electrólisis, distancia entre los electrodos, concentración de electrolito y pH de la solución. Emplearon una celda electroquímica simple con un ánodo y un cátodo, una solución de $40 \mathrm{mg} / \mathrm{L}$ de tartrazina con $400 \mathrm{mg} / \mathrm{L} \mathrm{de} \mathrm{NaCl}$ y DQO de $30 \mathrm{mg} / \mathrm{L}$. Se obtuvo como resultado la remoción casi del $100 \%$ de color y $90 \%$ de DQO; en condiciones de $\mathrm{pH}=5,78$, tiempo de electrolisis $=6$ minutos, densidad de corriente $=120 \mathrm{~mA} / \mathrm{cm}^{2}$, distancia entre electrodos $=1,5 \mathrm{~cm}$, con un ánodo de hierro y un cátodo de aluminio. Adicionalmente, compararon la eficiencia de la celda electroquímica con electrodos monopolares en serie y en paralelo y con electrodos bipolares. Los resultados obtenidos con los electrodos en configuración monopolar con conexión en serie fue la más eficaz. Concluyeron que el proceso con electrodo Fe/Al (ánodo/cátodo) fue más eficaz para el tratamiento, en comparación a los electrodos de Fe/Fe. En otro trabajo, Gupta et al., (2011) investigaron la degradación de la tartrazina mediante fotocatálisis heterogénea, con UV y $\mathrm{TiO}_{2}$ como catalizador.

Este proceso se llevó a cabo a diferentes condiciones de pH (2,2 a 11), dosis de catalizador (2 a $20 \mathrm{mg})$ y concentración del colorante (11-43 mg/L). Las condiciones óptimas encontradas para la degradación de 100 $\mathrm{mL}$ de solución a $30^{\circ} \mathrm{C}$, fueron: $32 \mathrm{mg} / \mathrm{L}$ de colorante, $\mathrm{pH}=11$ y $0,18 \mathrm{~g} / \mathrm{L}$ de $\mathrm{TiO}_{2}$ con un porcentaje de degradación del 93,6\% para la DQO y 96,1\% del colorante. Más recientemente, Oancea et al. (2013) evaluaron el proceso foto-Fenton para la degradación de tartrazina con una concentración de 5,53 $\mathrm{mg} / \mathrm{L}$ y $\mathrm{pH}=3$. Emplearon un fotoreactor a escala laboratorio y determinaron las mejores condiciones de operación para la concentración de $\mathrm{H}_{2} \mathrm{O}_{2}(6 \times 10-4 \mathrm{M}), \mathrm{Fe}^{2+}(8,28 \times 10-5 \mathrm{M})$ y temperatura $(323 \mathrm{~K})$. Después de 20 minutos de reacción, obtuvieron una degradación de la tartrazina del $98 \%$ y del COT del $43 \%$. Incrementando el tiempo de tratamiento a 120 minutos, reportaron una degradación del COT del $80 \%$. Otro avance importante fue el reportado por Thiam et al. (2014), quienes emplearon un tratamiento electroquímico secuencial que consistió en una etapa de electrocoagulación (EC) como pretratamiento y la posterior oxidación mediante proceso electro-foto-Fenton (EFF). Ellos realizaron el proceso de EC como pretratamiento empleando electrodos de Fe/Acero, con una concentración inicial de tartrazina de $278 \mathrm{mg} / \mathrm{L}$, $0,05 \mathrm{M}$ de $\mathrm{NaCl}, \mathrm{pH}=6,3$ y $200 \mathrm{~mA}$ para un tiempo de tratamiento de 15 minutos; posteriormente, centrifugaron la solución y el sobrenadante se sometió a un proceso de FEF, empleando electrodos de $\mathrm{DDB} /$ Electrodo de difusión de aire $(\mathrm{ADE})$ a un $\mathrm{pH}$ de 3 durante 300 minutos de reacción, obteniendo mineralización completa. En ninguno de los casos mencionados se reportó un análisis sistemático para encontrar las condiciones de operación óptimas.

En esta investigación se evaluó la viabilidad de los tres procesos de electro-oxidación mencionados (EO, EF y EFF) para la degradación y mineralización de la tartrazina. La eficiencia del proceso de EO fue evaluada y optimizada mediante la metodología de superficie de respuesta (MSR) (GilPavas et al., 2009). Se estudió cómo las variables más significativas para el proceso afectan la velocidad de degradación del colorante (DC) y de la materia orgánica e inorgánica en términos de la demanda química de oxígeno (DQO) y el grado de mineralización (en función de la degradación del carbono orgánico total, COT). La optimización mediante MSR implicó los siguientes pasos: (1) Implementación estadística del diseño de experimentos; (2) Estimación de los coeficientes de un modelo matemático utilizando técnicas de análisis de regresión; (3) Predicción de la respuesta y (4) Verificación del modelo. Se utilizó como herramienta un diseño del tipo BoxBehnken (DBB) para la programación de cada uno de los ensayos experimentales y la optimización de las condiciones de reacción. Posteriormente, EF y EFF fueron desarrollados a las condiciones óptimas de operación de la EO. 


\section{MATERIALES Y MÉTODOS}

\section{Muestras}

Muestras sintéticas fueron preparadas con agua desionizada y colorante tartrazina (89,33\% de pureza) suministrado por la empresa ColorQuímica. En la Tabla 1 se presentan la estructura química de la tartrazina, sus características y las condiciones iniciales de las muestras a tratar.

Tabla 1: Estructura química y características del colorante tartrazina

\begin{tabular}{|c|c|c|c|c|}
\hline \multicolumn{2}{|c|}{ Características } & \multicolumn{3}{|c|}{ Estructura Química } \\
\hline \multicolumn{2}{|c|}{$\begin{array}{l}\text { Formula Molecular: } \mathrm{C}_{16} \mathrm{H}_{9} \mathrm{~N}_{4} \mathrm{Na}_{3} \mathrm{O}_{9} \mathrm{~S}_{2} \\
\text { Clasificación química: azo } \\
\text { Numero C.I: } 19140 \\
\text { Apariencia: Amarillo } \\
\text { PM: } 534,385 \mathrm{~g} / \mathrm{mol}\end{array}$} & \multicolumn{2}{|c|}{$\mathrm{OH}_{\mathrm{N}}^{\mathrm{NaOOC}}$} & \\
\hline Parámetros & & \multicolumn{2}{|c|}{ Medición } & \\
\hline $\begin{array}{l}\text { Concentración de } \\
\text { tartrazina (mg/L) }\end{array}$ & 20 & 30 & 40 & 60 \\
\hline DQO (mg/L) & 27 & 40 & 59 & 77 \\
\hline $\mathrm{pH}$ & 6,08 & 6,09 & 6,1 & 6,09 \\
\hline Conductividad $(\mu \mathrm{S} / \mathrm{cm})$ & 1060 & 1060 & 1060 & 1060 \\
\hline Densidad $(\mathrm{g} / \mathrm{mL})$ & 1,001 & 1,001 & 1,001 & 1,001 \\
\hline Viscosidad (cP) & 1,002 & 1,002 & 1,002 & 1,002 \\
\hline
\end{tabular}

La adecuación de la conductividad se efectuó con $\mathrm{NaCl}$ grado comercial (Refisal) y el pH con $\mathrm{H}_{2} \mathrm{SO}_{4}$ al 99,1\% (Mallinckrodt Analytical Reagent) o NaOH grado comercial (Bell Chem Internacional S.A.). Para el proceso EF se utilizó $\mathrm{FeSO}_{4} .7 \mathrm{H}_{2} \mathrm{O}$ grado analítico (J.T. Baker) como fuente de iones $\mathrm{Fe}^{2+}$.

\section{Celda de Electro-Oxidación}

Para todos los experimentos, se empleó un reactor de vidrio de borosilicato con capacidad de $42 \mathrm{~mL}$. Este incluye dos electrodos con configuración monopolar: uno de placa plana de titanio (cátodo), con un área total $55 \mathrm{~cm}^{2}$ y área efectiva de reacción de $40 \mathrm{~cm}^{2}$; y el otro, un electrodo de diamante dopado con boro (ánodo DDB, 0,5 mm sustrato de silicona, marca Fraunhofer, USA Center for Coatings and Laser Applications) con un área total $6 \mathrm{~cm}^{2}$ y área efectiva de reacción de $2 \mathrm{~cm}^{2}$. Los electrodos fueron puestos verticalmente en la celda, con distancia entre ellos de $5 \mathrm{~mm}$. La celda contiene además un agitador magnético Corning.

Para el proceso de EFF, se usaron lámparas UV de 365 (4W). Se utilizó como fuente de energía un equipo BK-Precisión (0-30 V, 0-5 A) y un sistema de enfriamiento de flujo continuo conectado a un baño criogénico (Polyscience 712$)$, que permite mantener la temperatura de reacción constante $\left(25-30^{\circ} \mathrm{C}\right)$. En la Fig. 1 se muestra el diagrama de flujo del proceso.

El tiempo de reacción fue de 15 minutos para los ensayos experimentales y de 30 minutos para los estudios cinéticos. La eficiencia del proceso se evaluó midiendo los porcentajes de degradación del colorante (\%DC), de degradación de la DQO (\%DDQO) y mineralización de la solución (\%DCOT). Estos fueron calculados como se muestra en la expresión (7).

$\% D C=\frac{\left(C_{0}-C\right)}{C_{0}} * 100$

donde $C_{0}$ y $C$ representan, respectivamente, la concentración inicial y final del colorante, de la DQO o del COT. 


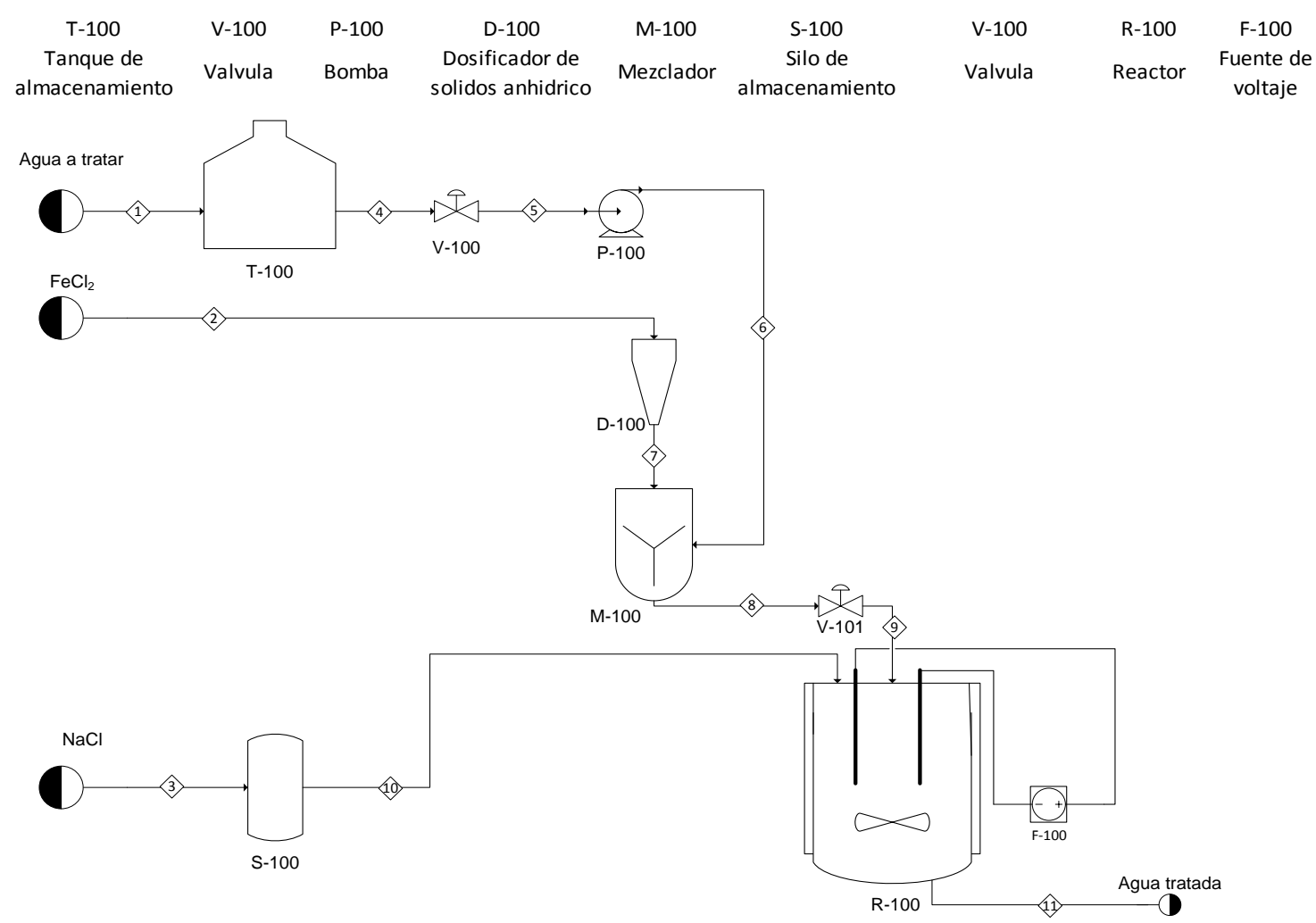

Fig. 1: Diagrama de flujo del sistema de electro-oxidación a escala de laboratorio

\section{Métodos Analíticos}

Para seguir la evolución de la cinética de degradación del colorante, se utilizó la técnica de espectroscopía (Espectrofotómetro, Spectronics Genesys 2PC). Las concentraciones se determinaron a partir de sus características de absorbancia en el UV-VIS $(200-700 \mathrm{~nm})$ y se midieron basados en la curva de calibración construida a la longitud de onda de máxima absorbancia $(430 \mathrm{~nm})$ con concentraciones de $10,15,20,30$, $40,50,60 \mathrm{mg} / \mathrm{L}$. El procedimiento empleado para medir la DQO fue el método estándar de reflujo cerrado, (método 5220D, Kit test 0-26, DQO 160, Macherey-Nagel). La medición del COT fue realizada usando el método estándar de oxidación húmeda con medición colorimétrica (método 5310D, Kit test 0-94, COT 60, Macherey-Nagel). Para este procedimiento se empleó un Termoreactor Spectrocuant TR 420 Merck y el equipo Nanocolor 500D Macherey-Nagel (Standar Methods, 2005). El pH y la conductividad de cada una de las muestras fueron determinadas mediante el equipo multiparamétrico ( $\mathrm{pHmetro} \mathrm{y} \mathrm{coductímetro)} \mathrm{Thermo}$ Scientific orion 5 star.

\section{Diseño experimental}

Se usó un diseño factorial fraccionado del tipo $2^{n-1}$, con tres puntos centrales. En la Tabla 2 se muestran los factores evaluados y los tres niveles elegidos para la programación de las pruebas experimentales.

Tabla 2: Variables diseño de experimentos preliminar. * La conductividad de la solución se modificó con diferentes concentraciones de $\mathrm{NaCl}(0,03 \mathrm{M}, 0,019 \mathrm{M}, 0,008 \mathrm{M})$

\begin{tabular}{|l|c|c|c|}
\hline \multicolumn{4}{|c|}{ Factores codificados $\mathrm{X}$} \\
\hline \multicolumn{1}{|c|}{ Variable } & -1 & 0 & 1 \\
\hline & Nivel 1 & Nivel 2 & Nivel 3 \\
\hline Concentración inicial de tartrazina $(\mathrm{Ci}, \mathrm{ppm})$ & 20 & 40 & 60 \\
\hline $\mathrm{pH}$ & 3 & 4,5 & 6 \\
\hline Conductividad ${ }^{*}(\mu \mathrm{S} / \mathrm{cm})$ & 945 & 2038 & 2927 \\
\hline Densidad de corriente $\left(\mathrm{mA} / \mathrm{cm}^{2}\right)$ & 5 & 10 & 15 \\
\hline Agitación $(\mathrm{rpm})$ & 240 & 290 & 340 \\
\hline
\end{tabular}


Se definió un diseño preliminar con 19 experimentos para determinar las variables más relevantes para el proceso de EO. De acuerdo con los resultados obtenidos (que no se presentan en este artículo), se fijaron los parámetros de conductividad y agitación de la solución en $1060 \mu \mathrm{S} / \mathrm{cm}$ y $340 \mathrm{rpm}$, respectivamente. Con estos valores fijos, se realizó un DBB que incluía las variables restantes: $\mathrm{pH}$, concentración de colorante y densidad de corriente. Los experimentos se realizaron en las condiciones mostradas en la Tabla 3.

Tabla 3: Variables de diseño experimentos Box-Behnken

\begin{tabular}{|c|c|c|c|}
\hline \multicolumn{4}{|c|}{ Factores codificados $X$} \\
\hline & -1 & 0 & 1 \\
\hline Variable & Nivel 1 & Nivel 2 & Nivel 3 \\
\hline Concentración inicial de tartrazina (Ci ppm) & 20 & 40 & 60 \\
\hline $\mathrm{pH}$ & 3 & 4,5 & 6 \\
\hline Densidad de corriente $\left(\mathrm{mA} / \mathrm{cm}^{2}\right)$ & 5 & 7,5 & 10 \\
\hline
\end{tabular}

Para el análisis estadístico se empleó el análisis de varianza (ANOVA), diagrama de Pareto, gráfica de superficie de respuesta y la correlación de los mismos, con el fin de determinar si cada uno de los factores analizados tiene un efecto significativo sobre la velocidad de degradación del colorante. Se realizaron 15 experimentos de manera aleatoria con el fin de evitar algún sesgo sistemático. Cada uno de los factores se estudió a tres niveles, de acuerdo con resultados obtenidos en ensayos preliminares. Las variables fueron codificadas de acuerdo a la ecuación (8) (Montgomery, 2005).

$$
X_{i}=\frac{\left(x_{i}-x_{p c}\right)}{\Delta x_{i}}
$$

donde $X_{i}$ es el nivel codificado, $x_{i}$ es el valor real, $X_{p c}$ es el valor real del punto central y $\Delta x_{i}$ es el valor del cambio de la variable entre nivel. Los datos fueron ajustados a un modelo polinómico de segundo orden. El modelo empleado en la superficie de respuesta fue el siguiente:

$$
Y_{i}=\beta_{0}+\sum_{1}^{3} \beta_{i} X_{i}+\sum_{1}^{3} \beta_{i i} X_{i i}^{2}+\sum_{1}^{3} \sum_{1}^{3} \beta_{i j} X_{i} X_{j}
$$

donde; $\beta_{0}, \beta_{i}, \beta_{i i}, \beta_{i j}$, son los coeficientes de regresión para los términos intercepto, lineal, cuadrático e interacciones, respectivamente y $X_{i}$ y $X_{J}$ son las variables independientes. La calidad de este modelo y su poder de predicción, están relacionados con el coeficiente de variación, $\mathrm{R}^{2}$. Las respuestas fijadas como objetivo en este proceso fueron el \%DC y el \%DDQO $\left(Y_{i}\right)$. La programación de los experimentos y análisis estadístico de los datos se realizó con ayuda del software estadístico Statgraphics 5.1.

\section{RESULTADOS Y DISCUSIÓN}

Los resultados de \%DC y \%DDQO obtenidos del diseño de experimentos para el proceso de EO se resume en la Tabla 4. Se observa que el mejor resultado fue bajo las condiciones de operación del ensayo 2, con un $\%$ DDQO del $77,5 \%$ y un \%DC del $96,9 \%$.

Tabla 4: Resultados del diseño de experimentos Box-Behnken para el proceso de EO

\begin{tabular}{|c|c|c|c|c|c|}
\hline Ensayo & $\mathrm{Ci}(\mathrm{ppm})$ & $\mathrm{i}\left(\mathrm{mA} / \mathrm{cm}^{2}\right)$ & $\mathrm{pH}$ & $\% \mathrm{DC}$ & $\%$ DDQO \\
\hline 1 & 40 & 7,5 & 4,5 & 91,42 & 61,97 \\
\hline 2 & 40 & 10 & 6 & 96,86 & 77,47 \\
\hline 3 & 40 & 7,5 & 4,5 & 89,62 & 60,56 \\
\hline 4 & 20 & 7,5 & 6 & 95,91 & 64,10 \\
\hline 5 & 20 & 10 & 4,5 & 97,61 & 66,67 \\
\hline 6 & 60 & 7,5 & 6 & 60,61 & 42,31 \\
\hline 7 & 20 & 7,5 & 3 & 96,81 & 58,97 \\
\hline 8 & 60 & 5 & 4,5 & 64,11 & 43,59 \\
\hline 9 & 20 & 5 & 4,5 & 96,31 & 76,92 \\
\hline 10 & 60 & 10 & 4,5 & 74,88 & 53,85 \\
\hline 11 & 40 & 5 & 6 & 97,21 & 67,61 \\
\hline 12 & 60 & 7,5 & 3 & 41,38 & 56,41 \\
\hline 13 & 40 & 7,5 & 4,5 & 91,77 & 73,24 \\
\hline 14 & 40 & 5 & 3 & 89,12 & 71,83 \\
\hline 15 & 40 & 10 & 3 & 79,49 & 70,42 \\
\hline
\end{tabular}


EI ANOVA de los resultados mostró que estadísticamente la Ci tiene un efecto muy significativo (valor de $\mathrm{P}<0,05)$ en el proceso de degradación de la materia orgánica. De igual manera, las interacciones Ci-Ci y i-i con un coeficiente de correlación de $\mathrm{R}^{2}=94,1 \%$ (Tabla 5).

Tabla 5: Análisis de la varianza ANOVA para el \%DDQO para el proceso de EO

\begin{tabular}{|c|c|c|c|c|c|}
\hline Fuente & Suma de cuadrados & $\begin{array}{c}\text { Grados } \\
\text { de } \\
\text { libertad }\end{array}$ & Cuadrado Medio & Razón-F & Valor-P \\
\hline $\mathrm{A}: \mathrm{Ci}$ & 621,281 & 1 & 621,281 & 34,8 & 0,002 \\
\hline$B: i$ & 8,92531 & 1 & 8,92531 & 0,5 & 0,5111 \\
\hline $\mathrm{C}: \mathrm{pH}$ & 4,72781 & 1 & 4,72781 & 0,26 & 0,6287 \\
\hline AA & 300,685 & 1 & 300,685 & 16,84 & 0,0093 \\
\hline$A B$ & 105,165 & 1 & 105,165 & 5,89 & 0,0596 \\
\hline$A C$ & 92,4482 & 1 & 92,4482 & 5,18 & 0,0719 \\
\hline BB & 199,92 & 1 & 199,92 & 11,2 & 0,0204 \\
\hline $\mathrm{BC}$ & 31,6969 & 1 & 31,6969 & 1,78 & 0,2401 \\
\hline $\mathrm{CC}$ & 23,9779 & 1 & 23,9779 & 1,34 & 0,2988 \\
\hline Error total & 89,2581 & 5 & 17,8516 & & \\
\hline Total (corr.) & 1521,59 & 14 & & & \\
\hline \multicolumn{6}{|l|}{$R^{2}=94,13 \%$} \\
\hline \multicolumn{6}{|c|}{$\mathrm{R}^{2}$ ajustado $=83,58 \%$} \\
\hline
\end{tabular}

Estos resultados se resumen en el diagrama de Pareto (Fig. 2). Así, es posible decir que la variable concentración de colorante (A:Ci) es la que más influye en el proceso de degradación (en \%DDQO).

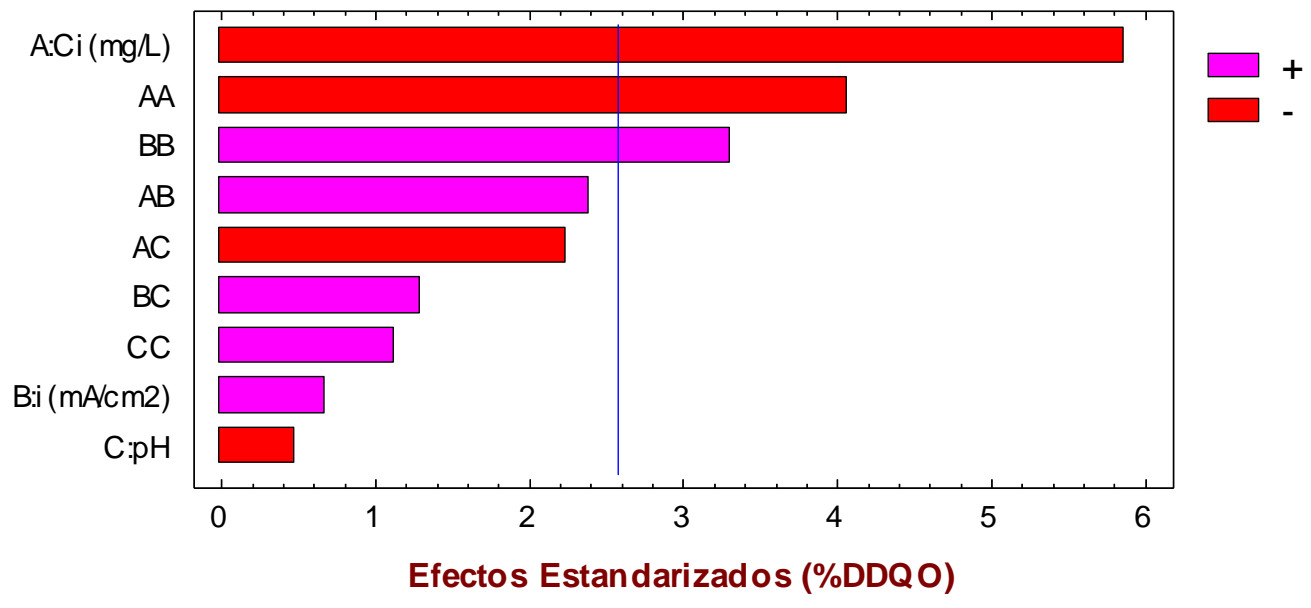

Fig. 2: Diagrama de Pareto. A: concentración de colorante, B: densidad de corriente y C: pH.

En la Fig. 3 se presenta la superficie de respuesta obtenida. Se observa que en el intervalo de Ci de 20 a 40 ppm, el \%DDQO es elevado y aumenta paulatinamente hasta aproximadamente 40 ppm. Después de este valor, el \%DDQO comienza a disminuir. De hecho, durante el proceso de EO se genera una cantidad significativa de radicales $\cdot \mathrm{OH}$, los cuales se encargan de la mineralización de la materia orgánica; la producción de radicales ${ }^{\circ} \mathrm{OH}$ bajo unas condiciones establecidas es constante, por lo tanto, al aumentar la concentración de colorante e incrementar la carga orgánica, la transformación del contaminante disminuye (como también lo observaron Ramírez et al., 2012).

$\% D D Q O=159.004+1.31621{ }^{*} \mathrm{C}_{\mathrm{i}}-24717.5 * \mathrm{i}-9.92583$ * $\mathrm{pH}-0.0225604$ * $\mathrm{C}_{\mathrm{i}}^{2}+$

$102.55^{*} \mathrm{C}_{\mathrm{i}}{ }^{*} \mathrm{i}-0.16025{ }^{*} \mathrm{C}_{\mathrm{i}}{ }^{*} \mathrm{pH}+1.17733 \mathrm{e}^{6}{ }^{*} \mathrm{i}^{2}+750.667^{*} \mathrm{i}^{*} \mathrm{pH}+1.13259$ * $\mathrm{pH}^{2}$ 


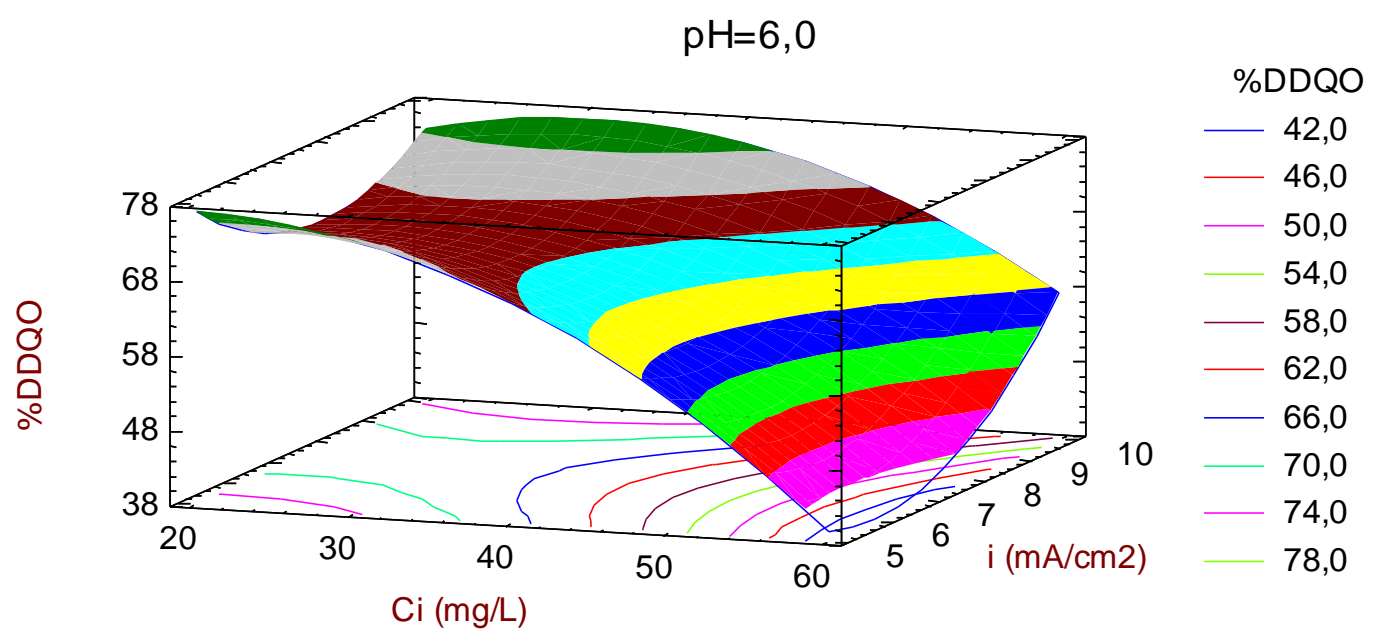

Fig. 3. Superficie de respuesta para el proceso de EO

El pH de la solución es otro factor importante en el tratamiento de aguas residuales. En los procesos de EO, algunos autores reportan que la degradación se favorece en medio básico (Rabaaoui y Allagui, 2012) mientras, en contraste, otros autores indican que la eficiencia del proceso se favorece en medio ácido (Panizza y Cerisola, 2004). De acuerdo a la revisión bibliográfica, se puede concluir, que el efecto del pH es fuertemente dependiente de la naturaleza del contaminante investigado y del soporte electrolítico empleado. Sin embargo, según la información incluida en la Tabla 4 y en la Fig. 2 no existe una influencia importante en el intervalo de $\mathrm{pH}$ estudiado (3-6). Con respecto a esta variable, finalmente es importante recalcar que el $\mathrm{pH}=6$ corresponde con el valor natural de varios efluentes industriales que contienen tartrazina, razón por la cual se seleccionará este valor para estudios posteriores.

La densidad de corriente afecta la eficiencia de degradación de los contaminantes, la cinética de reacción y los costos de operación del proceso de EO. La densidad de corriente incrementa y mejora la velocidad de degradación de los contaminantes debido a que la producción de radicales ${ }^{\circ} \mathrm{OH}$ aumenta. En la Fig. 3 se observa que el \%DDQO no varía significativamente en la región experimental evaluada (entre 5 y 10 $\mathrm{mA} / \mathrm{cm}^{2}$ ). Esto significa que la cantidad de radicales ${ }^{\circ} \mathrm{OH}$ y de $\mathrm{H}_{2} \mathrm{O}_{2}$ electro-generados es casi constante y suficiente para degradar totalmente la materia orgánica presente. Sin embargo, la naturaleza del contaminante y las condiciones de operación pueden generar conclusiones diferentes (Ramírez et al., 2012). Considerando los resultados obtenidos, las condiciones óptimas del proceso de EO fueron: concentración inicial de tartrazina $=30 \mathrm{ppm}$, densidad de corriente $=5 \mathrm{~mA} / \mathrm{cm}^{2}, \mathrm{pH}=6$, conductividad $=$ $1060 \mu \mathrm{S} / \mathrm{cm}$ (ajustada con $10 \mathrm{mM}$ de $\mathrm{NaCl}$ ), distancia entre electrodos $=5 \mathrm{~mm}$ y agitación de la solución a $340 \mathrm{rpm}$. A estas condiciones se realizó el estudio cinético.

\section{Cinética de Degradación de Tartrazina}

La Fig. 4a muestra la degradación de la tartrazina en diversos periodos de tiempo mediante el proceso de EO. De análisis espectrofotométricos, se ha establecido que las bandas a longitudes de onda de $257 \mathrm{~nm}$ y $430 \mathrm{~nm}$ son particulares para los anillos aromáticos individuales y al grupo cromóforo $-\mathrm{N}=\mathrm{N}$ - (responsable del color amarillo), respectivamente (Oancea y Meltzer, 2013). En la Fig. 4a se observa que, durante el proceso de EO, la banda de longitud de onda $430 \mathrm{~nm}$ disminuye considerablemente; sin embargo, la banda de $257 \mathrm{~nm}$ no presenta un cambio tan marcado. Luego de 10 min de tratamiento, se observa una disminución notable de la banda del grupo cromóforo. Sin embargo, se detecta la formación de compuestos intermedios, que registran una señal en el intervalo de longitud de onda entre 320-360 nm (en los tiempos de tratamiento de 15 a $20 \mathrm{~min}$ ). Al final del proceso de electro-oxidación (30 min), se observó una eliminación del color bastante notable. Se observó también que la señal asociada a la aparición de compuestos intermedios aumentó. Adicionalmente, se resalta la formación de un pico que sobresale en la longitud de onda de $220 \mathrm{~nm}$ y que corresponde a la presencia de $\mathrm{H}_{2} \mathrm{O}_{2}$ formado durante el proceso de EO. Observaciones similares fueron reportadas por Rajeshwar e Ibanez (1997). 


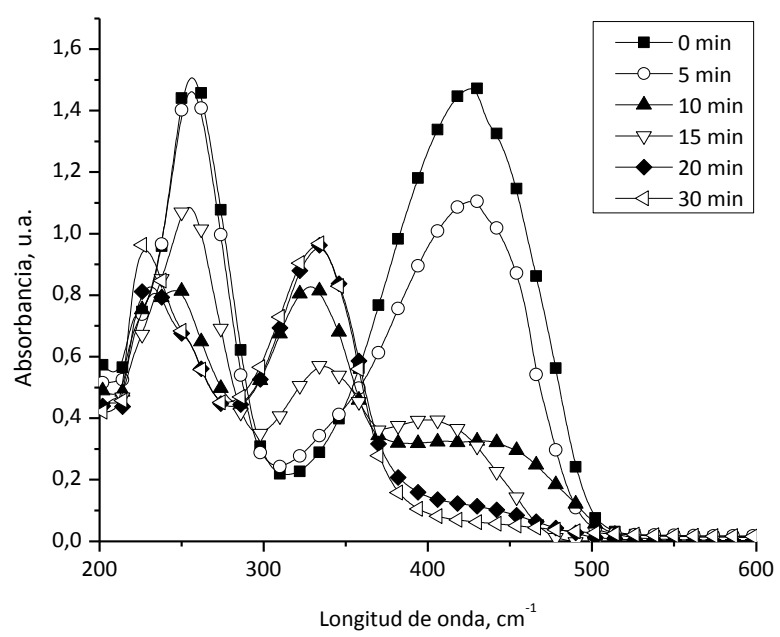

a)

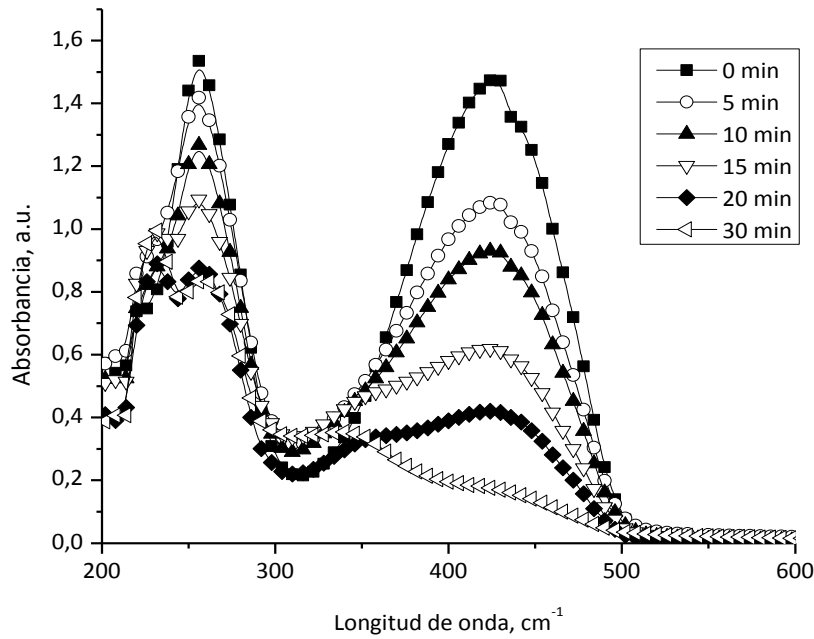

b)

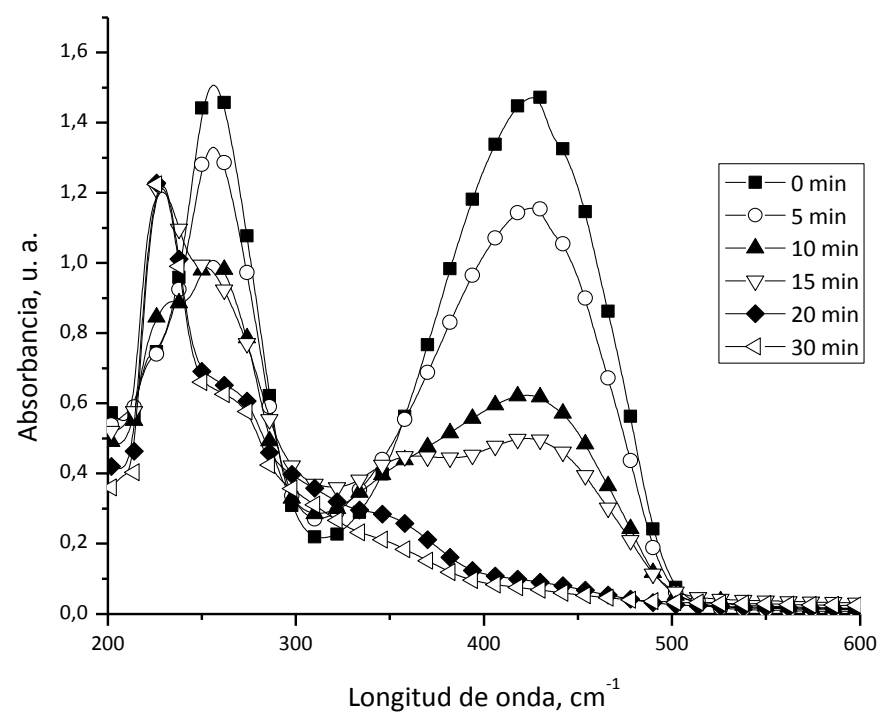

c)

Fig. 4: Cinética de degradación de la tartrazina mediante los procesos: a) EO, b) EF y c) EFF

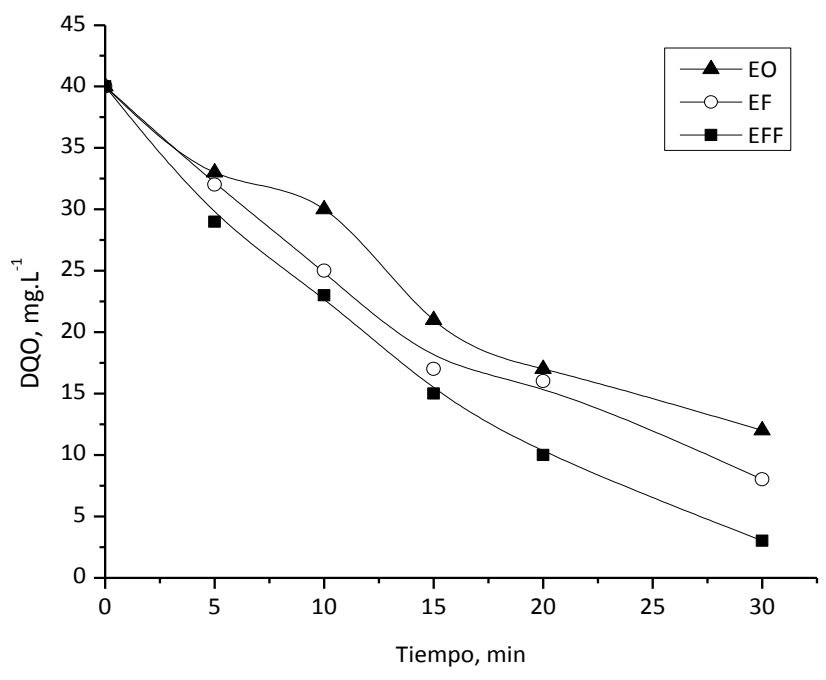

(a)

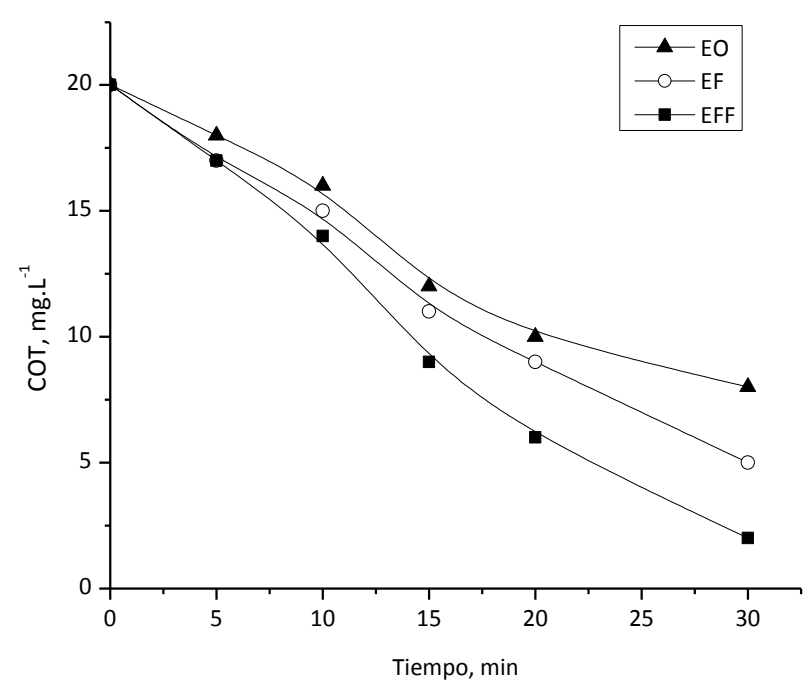

(b)

Fig. 5: Cinética de degradación de: a) DQO (a) y b) COT mediante los procesos de EO, EF y EFF 
Con el fin de mejorar el desempeño del proceso de EO, se adicionó a la mezcla reactiva $0,3 \mathrm{mM}$ de $\mathrm{Fe}^{2+}$ (valor previamente optimizado). Estas condiciones combinadas corresponden al proceso de electro-Fenton (EF). La Fig. 4b presenta los resultados obtenidos. Se observa que, durante los primeros 15 minutos, la degradación de colorante es más lenta que en el proceso EO. Sin embargo, el \%DC es similar al final de tratamiento. Es notable además que no se detectó la formación de compuestos intermedios (no hay señales en la región de longitud de onda entre 320-360 nm). Esto implica una mayor eficiencia del proceso de degradación denotándose un efecto sinérgico entre el $\mathrm{H}_{2} \mathrm{O}_{2}$ electro-generado y la adición de $\mathrm{Fe}^{+2}$, como también se encuentra reportado en otros trabajos (Costa et al., 2009; Cruz et al., 2012). Los resultados obtenidos con el proceso EFF se presentan en la Fig. 4c. Se observa que después de 30 minutos de tratamiento, la banda de $430 \mathrm{~nm}$ presenta el menor valor de los tres procesos evaluados. Adicionalmente, la banda a $220 \mathrm{~nm}$, y que corresponde al $\mathrm{H}_{2} \mathrm{O}_{2}$ formado, presenta un valor mucho más importante debido al efecto sinérgico que se presentan las condiciones del proceso, atribuibles al aumento del poder oxidativo de la reacción y la foto-descomposición de los contaminantes. En la Fig. 5 se muestra la evolución de la de degradación de DQO y COT. Se puede determinar que el proceso EFF es el más eficiente, con un \%DDQO de 92.5 y una mineralización (\%DCOT) del 90\%.

En general, los resultados obtenidos en esta investigación son bastante positivos para la eliminación de color (hasta un 100\%) y degradación y mineralización de la materia orgánica (92,5\%DDQO y 90\%DCOT). Es importante resaltar que las concentraciones iniciales de colorante empleadas en este studio fueron mayores que las analizadas por otros investigadores (Oancea y Meltzer, 2013; Gupta et al., 2011; Mordirshahla et al., 2007). En el trabajo realizado por Oancea y Meltzer (2013), utilizaron el proceso fotoFenton para la degradación de tartrazina, a una concentración de 5,5 ppm y un tiempo de operación 2 horas, obteniendo una mineralización del 80\%. El grupo de investigación de Mordirshahla et al., (2007) implementaron el proceso de electrocoagulación para tratar una solución de tartrazina de concentración de 40 ppm, obteniendo como resultado el 90\% de remoción de DQO (sin embargo, es de anotar que en este tipo de procesos se generan gran cantidad de lodos).

Por otro lado, Gupta et al., (2011), evaluaron la fotocatálisis heterogénea con $\mathrm{TiO}_{2}$ para la degradación de tartrazina con concentración de 32 ppm y obtuvieron una degradación de la DQO del 93,53\%. El único trabajo que supera la concentración evaluada en esta investigación es la efectuada por Thiam et al., (2014). Sin embargo, ellos requirieron implementar un proceso acoplado coagulación-EFF, donde en la etapa de coagulación remueven el $60 \%$ del COT y en la segunda etapa, proceso EFF, consiguen mineralizar completamente el colorante durante un tiempo de 300 minutos. Esto implica que en la primera etapa de tratamiento están transfiriendo el contaminante de una fase (líquida) a otra fase (sólida, proceso de centrifugación), con la subsecuente generación de lodos. Adicionalmente, requieren de mayores tiempos de electrólisis que el efectuado en esta investigación. Otro aspecto importante para resaltar, es que en ninguna de las investigaciones anteriores se empleó las técnicas de diseño estadístico de experimentos, herramienta fundamental para este tipo de investigaciones, para la optimización de recursos, tiempo, reactivos y las condiciones de operación del proceso.

\section{CONCLUSIONES}

Los procesos de degradación mediante electro-oxidación empleando electrodos de DDB se pueden aplicar de manera eficiente para la degradación de aguas contaminadas con el colorante tartrazina. En particular las siguientes conclusiones se pueden extraer a partir de los resultados de la investigación: (i) Con base en el análisis estadístico, se concluye que los parámetros más significativos en el proceso EO fueron: la concentración del colorante, la densidad de corriente y el pH de la solución. (ii) A partir de los resultados obtenidos (\%DC, \%DDQO y \%DCOT) mediante los procesos EO, EF y EFF se evidencia que estos tratamientos son favorables para la eliminación de tartrazina presente en el agua; adicional a la ventaja de requerir cortos tiempos de operación. Al comparar los resultados obtenidos de las cinéticas de degradación de los tres procesos, el proceso de EFF fue el más eficiente (\%DC=100, \%DDQO=93 y \%DCOT=90), presentando los mayores porcentajes de degradación. Esto dado que la reacción de Fenton, catalizada por la radiación UV, mejora la capacidad de formación de radicales hidroxilo $\left({ }^{\circ} \mathrm{OH}\right)$, los cuales promueven la oxidación de contaminantes y degradan la materia orgánica. En este proceso la producción de radicales ${ }^{\circ} \mathrm{OH}$ proviene de la acción combinada entre la oxidación anódica directa en el electrodo DDB y la reacción fotoFenton causada por el $\mathrm{H}_{2} \mathrm{O}_{2}$ generado por electrólisis del agua, el reactivo hierro $\mathrm{Fe}^{+2}$ y la radiación UV.

\section{AGRADECIMIENTOS}

Los autores agradecen a la Dirección de Investigación de la Universidad EAFIT, Medellín-Colombia, por el apoyo financiero para la realización de esta investigación. 


\section{REFERENCIAS}

Badellino, C., C. Arruda y R. Bertazzoli, Oxidation of pesticides by in situ electrogenerated hydrogen peroxide: Study for the degradation of 2,4-dichlorophenoxyacetic acid, Journal of Hazardous Matererials: 137, 856-864 (2006).

Brillas, E. y otros tres autores, Electrochemical incineration of diclofenac in neutral aqueous medium by anodic oxidation using Pt and boron-doped diamond anodes, Chemosphere: 79, 605-612 (2010).

Costa, C. y otros tres autores, Electrochemical oxidation of acid black 210 dye on the boron-doped diamond electrode in the presence of phosphate ions: Effect of current density, $\mathrm{pH}$, and chloride ions, Electrochimica Acta: 54, 7048-7055 (2009).

Cruz, K. y otros cinco autores, Optimization of electro-Fenton/BDD process for decolorization of a model azo dye wastewater by means of response surface methodology, Desalination: 286, 63-68 (2012).

Cruz, K. y otros seis autores, Determination of optimum operating parameters for Acid Yellow 36 decolorization by electro-Fenton process using BDD cathode, Chemical Engineering Journal: 160, 199-206 (2010).

Esquivel, K. y otros cuatro autores, Development of a $\mathrm{TiO}_{2}$ modified optical fiber electrode and its incorporation into a photoelectrochemical reactor for wastewater treatment, Water Research: 43, 3593-3603 (2009).

Flox, C. y otros seis autores, Solar photoelectro-Fenton degradation of cresols using a flow reactor with a boron-doped diamond anode, Applied Catalysis B: Environmental: 75, 17-28 (2007).

GilPavas, E., I. Dobrosz-Gómez y M. Á. Gómez-García, Decolorization and mineralization of Diarylide Yellow 12 (PY12) by photo-Fenton process: the Response Surface Methodology as the optimization tool, Water Science \& Technology: 65, 1795-1800 (2012).

GilPavas, E. y otros cuatro autores, The Box-Behnken experimental design for the optimization of the electrocatalytic treatment of wastewaters with high concentrations of phenol and organic matter. Water Science \& Technology: 60, 2809-2818 (2009).

Gupta, V. K. y otros cuatro autores, Removal of the hazardous dye-Tartrazine by photodegradation on titanium dioxide surface, Materials Science and Engineering: 31, 1062-1067 (2011).

Jonstrup, M., M. Punzi y B. Mattiasson, Comparison of anaerobic pre-treatment and aerobic post-treatment coupled to photo-Fenton oxidation for degradation of azo dyes. Journal of Photochemestry and Photobiology: 224, 55-61 (2011).

Martínez-Huitle, C.A. y E. Brillas. Decontamination of wastewaters containing synthetic organic dyes by electrochemical methods: A general review, Applied Catalysis B: Environmental, 87, 105-145 (2009).

Modirshahla, N., M. Behnajady y S. Kooshaiian, Investigation of the effect of different electrode connections on the removal efficiency of Tartrazine from aqueous solutions by electrocoagulation, Dyes and Pigments: 74, 249-257 (2007).

Mohan, N., N. Balasubramanian, y C.A. Basha, Electrochemical oxidation of textile wastewater and its reuse, Journal of Hazardous Materials: 147, 644-651 (2007).

Montgomery, D.C., Design and Analysis of Experiments, John Wiley \& Sons, Hoboken, New Jersey, Estados Unidos de América, (2005).

Oancea, P. y V. Meltzer, Photo-Fenton process for the degradation of Tartrazine (E102) in aqueous medium, Journal of the Taiwan Institute of Chemical Engineers: 44, 990-994 (2013).

Oturan, M.A. y otros cuatro autores, Kinetics of oxidative degradation/mineralization pathways of the phenylurea herbicides diuron, monuron and fenuron in water during application of the electro-Fenton process, Applied Catalysis B: Environmental, 97, 82-89 (2010). 
Panizza, M. y G. Cerisola, Application of diamond electrodes to electrochemical processes, Electrochimica Acta: 51, 191-199 (2005).

Patiño, K., Arroyave, S., Marín J. Oxidación Electroquímica y Ozonización Aplicadas al Tratamiento de Aguas de Lavado de la Producción de Biodiesel, Información tecnológica: 23(2), 41-52 (2012).

Rabaaoui, N. y M.S. Allagui, Anodic oxidation of salicylic acid on BDD electrode: variable effects and mechanisms of degradation, Journal of Hazardous Materials: 243, 187-192 (2012).

Rajeshwar K. y J. Ibanez, Environmental Electrochemistry: Fundamentals and Applications in Pollution Abatement. Academic Press, Los Angeles, California, Estados Unidos de América (1997).

Ramírez, C. y otros siete autores, Electrochemical oxidation of methyl orange azo dye at pilot flow plant using BDD technology, Journal of Industrial and Engineering Chemistry:19, 571-579 (2012).

Robinson, T. y otros tres autores, Remediation of Dyes in Textile Effluent: A Critical Review on Current Treatment Technologies with a Proposed Alternative, Bioresource Technology: 77, 247-255 (2001).

Rocha, E.M.R. y otros cuatro autores, Landfill leachate treatment by solar-driven AOPs, Solar Energy: 85, 46-56 (2011).

Standard methods for the examination of water and waste water, $20^{\mathrm{a}}$ Edición (Centenial edition), American Public Health Association (APHA). Washington, Estados Unidos de América (2005).

Thiam, A., Zhou, M., Brillas, E., Sirés, I. Two-step mineralization of Tartrazine solutions: Study of parametersand by-products during the coupling of electrocoagulation withelectrochemical advanced oxidation processes. Applied Catalysis B: Environmental, 150-151, 116-125 (2014).

Wawrkzkiewics, M. y Z. Hubicki, Removal of tartrazine from aqueous solutions by strongly basic, Journal of Hazardous Materials: 164, 502-509 (2009).

Yusuf, Y. y S. Reza, Anodic oxidation of Reactive Black 5 dye using boron doped diamond anodes in a bipolar trickle tower reactor, Separation and Purification Technology: 85,130-136 (2012). 